\title{
Heparin Coating Technology in Cardiopulmonary Bypass
}

\author{
Kun Zhang ${ }^{1}$,Rui Xue ${ }^{2, *}$ and Shuwei Shen ${ }^{1}$ \\ ${ }^{1}$ Department of Anesthesiology, Jingzhou Central Hospital, The Second Clinical Medical College, Yangtze University, \\ Jingzhou, Hubei Province,434020, China. \\ ${ }^{2}$ Department of Anesthesiology, Renmin Hospital, Hubei University of Medicine, Shiyan, Hubei, China. \\ Corresponding Email: 7885909@qq.com
}

\begin{abstract}
Heparin coating technology has become increasingly mature, which is conducive to the material of blood, biocompatibility proved very important, therefore, heparin coating technique has become a development cycle is very useful for in vitro selection of patients. On the other hand, the overall cost of medical treatment will increase because of the use of heparin coated materials and devices, so the clinical use of heparin coated materials is limited. In this paper, the principle of heparin coating technology, heparin coating technology should be applied in the norms of the external circulation, and related heparin coating technology development status analysis.
\end{abstract}

Keywords. Cardiopulmonary bypass, heparin coating technology, principle.

\section{Introduction}

The researches have proved that there is the heparin-like molecular structure (heparin sulfate) in a microvascular endothelial cell; when the solidification state of blood changes, such endothelial cells will militate [1-3]. Therefore, it may after all be accepted as a reasonable method to modify the abiotic surface with heparin substances to make it to have high blood compatibility. Heparin solidification, which has been widely studied on the blood contact surface of CPB equipment or for heparin coating, has been currently regarded as the most promising method to improve the biocompatibility.

\section{Principle of heparin coating technology}

As shown in the past researches, the heparin coating surface is formed by enhancing the catalysis of antithrombin-III and reducing thrombus. Although the favorable side of the technology has not been proven yet, the latest researches show that the advantage of heparin coating technology is the selective attachment of plasma proteins, which can rapidly form a pseudomembrane of blood compatibility on the surface of material and, meanwhile, can prevent albuminous degeneration from enhancing the activity of attachment protein and blood cell [4]. It is also found through related researches that the plasma proteins, such as blood coagulation factor XII, fibrinogen and transparent binding protein, on the surface of artificial material can enhance the anticoagulant activity.

\section{Principles of heparin coating technology for cpb equipment}


Universal surface principle: its representative method is to combine polyethyleneimine (PEI) with the surfaces of all sorts of materials by means of sedimentation or ionic bond so that all the material surfaces have affinity with the heparin degraded with nitrous acid.

Universal coating principle: it is to process heparin with binding agent so that its physico-chemical property can be changed; then, the heparin will have high affinity with the surfaces of all sorts of materials and its anticoagulation property can be kept simultaneously. Duraflo II heparin is namely an ideal universal coating applicable to heparin coating of CPB equipment. After it is diluted to the concentrated needed with organic solvent, 1, 1, 2trichloro-1, 1, 2fluoro-ethane, it is injected into the human's CPB loop for circulation, is emptied and is dried for standby. The coating will not cracks or expand under stress and the residual solvent can be easily drained.

\section{Heparin coating technology in $\mathrm{cpb}$}

The heparin covalent bond coating technology can be divided into three types: ionic bond combination; covalent bond combination and fixed end point. Its molecular formula is shown in Figure1.

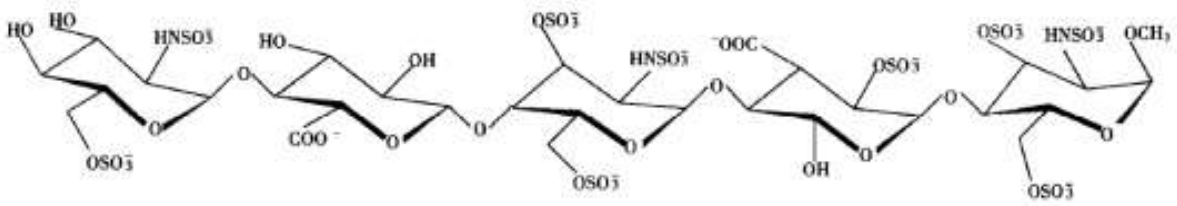

Fig.1. Schematic diagram for molecular structure of Heparin.

The heparin with its ionic bond combination carries a lot of negative electricity. The positive ion inducer is used to lead positive ions to the surface of synthetic material and attracts heparin with static electricity. The benzylamine-heparin with benzylamine as its inducer has been widely used in clinic but is not applicable to CPB because it is very easily soluble in normal saline [5-7]. TDMAC-heparin (hydrochloric acid 3-12 alkyl methylamine - heparin) is a very practical heparin coating material synthesized by Grode and the shunting pipes with TDMAC-heparin are available now. The combination between some positive ion genes, such as N-N dimethylamide vinyl, and polymer substrate can also help the heparin attachment. After the complexation of poly ammonia 1 and heparin, it can adhere to polyurethane and glass surface.

The mode of covalent bond combination is to fix heparin by using the functions of - CNS radical, -NH2 radical, -OH radical and other special active groups on the surface of high polymer material and the $-\mathrm{OH}$ radical of heparin molecule [8]. As heparin has plenty of functional groups which are combined with the surface of material excessively firmly, resulting in the loss of activity in heparin molecule, the expected anticoagulation effect usually cannot be reached.

The mode of fixed end point is firstly to prepare a binding ion complex on the surface of biomaterial; then, activate the heparin through a controlled nitrous acid degradation step so that the heparin molecule can form the final terminal free aldehyde group. This heparin and the glutamic acid dialdehyde can have a chemical reduction reaction through the ammoniation reducing action so as to fix heparin in the mode of covalent bond on the surface of material containing amino $[9,10]$. It's worth noting that part of such heparin molecules will degrade to lower the catalytic activity in solution. However, because of its very proper binding site between such heparin molecules and the surface of biomaterial, this mode can truly generate the anticoagulation coating materials.

\section{Conclusion}

One's whole body needs to be heparinized in the CPB operation. The heparin modification to CPB lines can make them to have better biological compatibility. This coating method, which can lead to relatively secure heparin binding within 8 hours allowed by an CPB operation, is applicable to clinics. To sum up, the heparin binding technology, which is a newly developed technology in recent years, can improve CPB lines and the biological compatibility of blood and can reduce the inflammatory reaction incurred by contacting the surface of a foreign matter. 


\section{References}

1. Gravlee GP.Heparin-coated cardiopulmonary bypass circuits.Journal of cardiothoracic and vascular anesthesia. (1994) Apr; 8 (2 ):213-22.

2. Stammers AH, Christensen KA, Lynch J, Zavadil DP .Quantitative evaluation of heparin-coated versus non-heparin-coated bypass circuits during cardiopulmonary bypass. The journal of extra-corporeal technology 1999 Sep; 31 (3 ):135-41.

3. Ovrum E, Tangen G, Oystese R, Ringdal MA, Istad R .Comparison of two heparin-coated extracorporeal circuits with reduced systemic anticoagulation in routine coronary artery bypass operations. The Journal of thoracic and cardiovascular surgery $2001 \mathrm{Feb} ; 121$ (2):324-30.

4. Van Poucke S, Stevens K, Wetzels R, Kicken C, Verhezen P, Theunissen M, Kuiper G, van Oerle R, Henskens Y, Lancé M.Early platelet recovery following cardiac surgery with cardiopulmonary bypass.Platelets. 2016 May 10:1-7.

5. Olsson A, Alfredsson J, Hkansson E, Svedjeholm R, Berglund J, Berg S.Protamine reduces whole blood platelet aggregation after cardiopulmonary bypass.Scand Cardiovasc J. 2016;50(1):58-63.

6. Kehara H, Takano T, Ohashi N, Terasaki T, Amano J.Platelet function during cardiopulmonary bypass using multiple electrode aggregometry: comparison of centrifugal and roller pumps.Artif Organs. 2014 Nov;38(11):924-30.

7. Ortmann E, Klein AA, Sharples LD, Walsh R, Jenkins DP, Luddington RJ, Besser MW.Point-of-care assessment of hypothermia and protamine-induced platelet dysfunction with multiple electrode aggregometry (Multiplate) in patients undergoing cardiopulmonary bypass.Anesth Analg. 2013 Mar;116(3):533-40.

8. Rubens FD.Cardiopulmonary bypass technology transfer: musings of a cardiac surgeon.Journal of biomaterials science. Polymer edition $2002 ; 13$ (4):485-99.

9. Chryssos A, Stroup SJ , Pifer MM , Tawil M,Conrad CG .Management of Heparin-Resistant Patients with Benefits? Maximizing Biocompatibility in Cardiopulmonary Bypass: Combining ATryn Recombinant Antithrombin III and Carmeda Heparin-Bonded Perfusion Circuits: A Case Series.The journal of extra-corporeal technology2015 Mar; 47 (1 ):44-7.

10. Zhang R, Huang Y, Zhang M, Liu J.Bivalirudin utilization in rats undergoing cardiopulmonary bypass: preventing the increase of antiheparin/platelet factor 4 antibody in perioperative period.Clin Appl Thromb Hemost. 2014 Mar;20(2):196-8. 